Monika Guerra-Glarner*

\title{
Le commentaire de Tankuang sur l'Eveil à la Foi dans le Grand Véhicule: la probable influence de Wonhyo
}

DOI 10.1515/asia-2016-0056

Abstract: Tankuang was a Chinese monk active during the eighth century. His texts were found in Dunhuang at the beginning of the twentieth century, and only then included in the Taishō canon. Tankuang wrote several commentaries on numerous Mahāyāna sutras, and two of these texts are commentaries on the Dasheng qixin lun. This paper focuses on the influence of two Korean monks, Wonch'uk and Wonhyo, on Tankuang's commentaries. Whereas it seems clear that Tankuang was affiliated to the Ximing lineage of Wonch'uk, there is no such evidence concerning the influence of Wonhyo. But it appears that Tankuang is taking over some of the concepts introduced by Wonhyo, and there are some clear cases of textual and doctrinal evidence of this influence that I will present in this paper.

Keywords: China, Dunhuang manuscript, Mahāyāna Bouddhism, Yogācāra, Wonhyo

\section{Introduction}

Tankuang est un moine originaire du Nord-Ouest de la Chine, dont les écrits, retrouvé à Dunhuang au début du $20^{\text {ème }}$ siècle, font aujourd'hui partie du canon bouddhique sino-japonais. Après avoir effectué une partie de ses études religieuses à Chang’an, au monastère de Ximing, il séjourna dans plusieurs monastères du Nord-Ouest de la Chine et termina sa vie à Dunhuang, à l'époque de la domination tibétaine.

Il a écrit plusieurs commentaires à des sūtra du Grand Véhicule, dont deux sur le Dasheng Qixin Lun $(Q X L) .{ }^{1}$ Le premier fut écrit aux alentours de 761 en cinq

1 Texte important du Mahāyāna, le 大乘起信論 ( Eveil à la Foi dans le Grand Véhicule ») consiste en une habile synthèse des principales thèses mahāyānistes parvenues en Chine à cette époque. Originellement attribué à Aśvaghoṣa, il est aujourd'hui largement admis qu'il s'agit

*Corresponding author: Monika Guerra-Glarner, Unité des études chinoises, Université de Genève, Rue de Candolle 2, 1211 Geneva 4, Switzerland. E-mail: Monika.Guerra@etu.unige.ch 
fascicules, dont deux sont aujourd'hui perdus. ${ }^{2}$ Dans ce commentaire très détaillé, Tankuang compare les deux « traductions » du QXL, celle attribuée à Paramārtha, datant du milieu du $6^{\text {ème }}$ siècle, et la deuxième, effectuée par Śikșānanda dans le courant du $7^{\text {ème }}$ siècle.

L’identité des maîtres de Tankuang durant son séjour au monastère Ximing environ un siècle après Xuanzang ainsi que les influences qu'il a subies ne sont pas connues. ${ }^{3}$ Mais c'est là qu'il se familiarisa avec les textes Yogācāra, et probablement également avec les écrits de Wonch'uk ${ }^{4}$ (613-696), qui avait résidé dans ce monastère, et en avait été abbé, quelques années auparavant.

Il est aujourd'hui avéré qu'il existait au moins deux courants parallèles de l'école Faxiang ${ }^{5}$ en Chine du Nord; le courant « orthodoxe » de Kuiji ${ }^{6}$ (632-682), et le courant « hérétique » introduit par Wonch'uk. Mon hypothèse de départ est celle d'une affiliation de Tankuang au second courant, dont les enseignements semblent avoir survécu à la rébellion d'An Lushan, et auraient été diffusés à Dunhuang par l'intermédiaire de Tankuang en même temps que le célèbre commentaire de Wonch'uk sur le Samidinirmocana Sūtra. ${ }^{7}$

d'un apocryphe composé directement en chinois, vers le milieu du $6^{\text {ème }}$ siècle. Il a été commenté par les plus éminents commentateurs, tels que Huiyuan, Wonhyo et Fazang. La question de l'origine du Traité reste ouverte et est toujours discutée par les spécialistes, en particulier au Japon.

2 Le Dasheng Qixin Lun guangshi 大乘起信論廣釋 (Commentaire général du Traité de l'Eveil de la Foi dans le Grand Véhicule, T. 2814).

3 Nous ne savons pas grand-chose de la vie de Tankuang. Bien qu'il fut l'auteur de volumineux commentaires et de lectures publiques, aucune de ses œuvres ne fut incluse dans le canon bouddhique durant les siècles qui suivirent et son nom ne fut pas non plus mentionné dans les Biographies de moines éminents. La raison en est probablement que ses activités furent confinées à la région proche de Dunhuang, et que ses travaux n'ont pas circulés jusqu'à Chang'an. En effet, la période de composition des œuvres de Tankuang est une époque troublée de l'histoire chinoise, dominée par deux événements importants; la Rébellion d'An Lushan, qui plongea la Chine dans le chaos durant de longues années entre 755 et 763 , et l'occupation durant presque un siècle (762-848) d'une grande partie de la Chine située sur la rive ouest du Fleuve jaune par les rois tibétains. Le peu que nous connaissons de la vie de Tankuang provient de la préface de l'un de ses commentaires (T. 2812), de laquelle on peut déduire qu'il est né à Jiankang (健康, garnison militaire située à l'est de Dunhuang) au tout début du $8^{\text {ème }}$ siècle. Nous savons par d'autres sources qu'il est probablement mort entre 781 et 788.

4 Yuance 圓測 en chinois.

5 Le concept d'« école Faxiang » a été interprété comme étant le Yogācāra orthodoxe qui succéda aux enseignements Yogācāra de Xuanzang et à sa suite toute la nouvelle tradition Yogācāra de l'Asie de l'est, qui est basée sur les traductions des nouveaux textes canoniques. 6 窅基, également appelé 《 maître Ci’en » (ci'en dashi 慈恩大師), Dacheng ji 大乘基 ou simplement $J i$ 基.

7 Cho 2005: 193. 
La seconde hypothèse que j'aimerais également vérifier et que je désire présenter dans cet article est celle de l'influence d'un autre moine coréen sur le commentaire de Tankuang. Bien que parmi les commentaires du QXL disponibles au début de la dynastie Tang, celui de Fazang ${ }^{8}$ était vraisemblablement le plus connu, il semble que Tankuang s’intéressa plus encore à ceux de Wonhyo (617-686). ${ }^{9}$ En effet, mis à part le fait que tous deux citent fréquemment les mêmes sūtra du Grand Véhicule, il semble que Tankuang connaissait très bien ses commentaires et qu'il a également repris de nombreux concepts introduits par ce dernier.

Enfin, je vais présenter quelques évidences textuelles qui semblent indiquer que Tankuang s'est servi des textes de Wonhyo lors de la rédaction de ses propres commentaires.

\section{2 «Ancien » Yogacāra et « nouveau » Yogacāra}

Les histoires $d u$ bouddhisme chinois distinguent en général deux étapes dans le développement du bouddhisme Yogācāra en Chine. ${ }^{10}$ L'interprétation introduite par Paramārtha (499-569), ${ }^{11}$ qui traduisit un grand nombre de textes bouddhistes indiens qu'il avait emmenés avec lui. Ses principales traductions étaient basées sur des textes indiens de Yogācāra, qui devinrent la base de l'école Shelun. ${ }^{12}$

Un siècle plus tard, la nouvelle série de traductions effectuées par Xuanzang (602-664) et son équipe inaugura la deuxième transformation majeure dans le développement doctrinal du bouddhisme Yogācāra chinois. Ce groupe de textes fut appelé " nouvelles traductions ", en contraste avec les " anciennes traductions » de Paramārtha. La création du Cheng weishi lun $(C W S L)^{13}$ marque le début

8 T.1846.

9 Yuanxiao 元曉 en chinois. Il est l'auteur de deux commentaires du QXL (T.1844 et T.1845).

10 Cf. Paul 1984.

11 Moine indien arrivé en Chine par les routes maritimes du sud en 546.

12 Ainsi nommée d'après le texte qu'il traduisit, le She Da-sheng lun (Mahāyāna-samgrāha ou "Somme du Grand Véhicule » d'Asanga).

13 L' «Établissement du rien-que-connaissance » (T. 1585). Il s'agit d'une compilation synthétique, établie et traduite par Xuanzang en 659-660, des enseignements de l'école Yogācāra, d'après les commentaires de dix maîtres indiens de l'école sur la «Trentaine » (Triṃśikā, T.1586), un petit traité en trente stances et en prose, dans lequel Vasubandhu avait condensé sa doctrine. Il s'agit de la seule traduction de Xuanzang qui n'est pas une traduction directe d'un texte, mais plutôt un tableau sélectif et éditorial de différents textes distincts qui peut être vu comme représentatif de sa position générale sur la doctrine. 
de la deuxième tradition, et c'est également sur elle que se fonde l'école Faxiang.

Kuiji, considéré comme le successeur de Xuanzang, aurait complètement répudié les enseignements Yogācāra introduits par Paramārtha un siècle plus tôt, et promu à leur place ceux développés par Xuanzang. En fait, en établissant ses fondements théologiques, Kuiji fut le fondateur effectif de l'école Faxiang, et celui qui l'a différenciée de la compréhension préalable du Yogācāra.

Une sorte de bifurcation aurait donc eu lieu dans la tradition Yogācāra en Asie de l'est, entre un « ancien » Yogācāra, basé sur les enseignements de Paramārtha, et un " nouveau » Yogācāra, fondé sur ceux de Xuanzang, considérés comme antagonistes sur le plan doctrinal.

Durant les $6^{\text {ème }}$ et $7^{\text {ème }}$ siècles, les exégètes bouddhistes est-asiatiques considéraient généralement cette distinction entre les deux systèmes comme un véritable antagonisme doctrinal entre les défenseurs de la théorie selon laquelle tous les êtres deviennent des buddha (yiqie jie cheng 一切皆成) ou théorie du tathāgatagarbha, ${ }^{14}$ et ceux qui distinguaient cinq lignages distincts (wuxing gebie 五性各別). ${ }^{15}$ Cette controverse est généralement connue sous le nom de " controverse de la Nature de Buddha ». Cette interprétation a également influencé la recherche moderne, qui a intégré cette dichotomie historique entre un « ancien » et un « nouveau » système Yogācāra.

La recherche récente tend cependant à remettre en question cette dichotomie traditionnelle ainsi que l'idée d'une opposition entre les vues de Paramamārtha et celles de Xuanzang. ${ }^{16}$ Certains travaux montrent une connexion possible entre Paramārtha et Xuanzang par le biais de l'étude d'archives historiques concernant les " nouvelles écoles », suggérant que Xuanzang luimême ne se serait pas prononcé contre les vues de Paramārtha. C’est Fazang

14 Rulaizang 如來藏 《Embryon de tathāgata ou Nature de Buddha ». La théorie du tathāgatagarbha fut clairement exposée pour la première fois vers 200 apr. J.-C., dans le Tathāgatagarbha-sūtra et le Śrīmālādevī Simhanāda Sūtra (le « Sūtra du rugissement du lion adressé à la reine Śrīmālādevī »). L’idée du tathāgatagarbha: tout être animé a la capacité d'atteindre le plein Eveil d'un Buddha parce que le tathāgatabarbha existe chez tous sans aucune exception. En tant qu'êtres vivants, ils sont tous des embryons de buddha (Cornu 2006: 610-611).

15 Nous allons revenir sur cette théorie plus en détails un peu plus loin.

16 KENG Ching propose dans sa thèse la possibilité que Paramārtha et Xuanzang aient pu être d'accord sur de nombreux points doctrinaux, affirmant que l'image préexistante de Paramārtha comme défenseur de la théorie tathāgatagarbha est une vue retrospective erronée qui nous vient de Tanqian (542-607), un disciple tardif de Paramārtha (Keng 2009). 
qui, le premier, aurait comparé les théories Tathāgatagarbha et Yogācāra dans une forme doctrinale systématique, en leur attribuant deux niveaux séparés d'enseignements. ${ }^{17}$

Le fait, avéré d'un point de vue historique, qu'il existait des contrastes entre les exégètes de l' " ancien » et du " nouveau » Yogācāra ne signifie donc pas que toute la tradition du nouveau Yogācāra était entièrement opposée à l'ancien Yogācāra, et il est donc impossible de réduire toute la tradition du « nouveau » Yogācāra en une seule entité antagoniste d'un point de vue doctrinaire à l' " ancien » Yogācāra. Bien que Kuiji, un des disciples majeurs de Xuanzang, ait systématisé les enseignements Yogācāra de la littérature bouddhique nouvellement introduite, il existait en effet d'autres lignes doctrinales que la sienne dans la tradition du "nouveau " Yogācāra. ${ }^{18}$ Ainsi Wonch'uk, un autre disciple majeur de Xuanzang, appartenant également au groupe du « nouveau » Yogācāra, défendit-il des positions doctrinales différentes de Kuiji. Les chercheurs modernes s'accordent en général sur le fait que Kuiji et Wonch'uk appartiennent à différents lignages scolastiques, connu sous le nom de lignage Ci'en 慈恩 et lignage Ximing 西明, ${ }^{19}$ d'après le nom des monastères auxquels ils appartenaient.

\section{La controverse sur la Nature de Buddha et la question des icchantika}

La distinction doctrinale la plus saillante entre la position Tathāgatagarbha et la position Yogācāra de Kuiji se trouve dans leurs perspectives différentes concernant

$17 \mathrm{Au}$ contraire, certaines recherches suggèrent qu'il ne s'agissait pas de deux systèmes doctrinaux antagonistes, mais étaient associés l'un à l'autre dès l'époque de Vasubandhu. KENG affirme par exemple que Vasubandhu avait, déjà à son époque, intégré la notion de tathāgatagarbha, bien que son acceptation de la notion de tathāgatagarbha ne comporte pas exactement les mêmes connotations que celle de la théorie Tathāgatagarbha que l'on trouve dans des travaux tels que le QXL. Keng 2009: 401-404.

18 Certains spécialistes japonais ont également soulevé des questions quant à l' " orthodoxie » de la position Yogācāra de Kuiji dans la «nouvelle » tradition Yogācāra (Lee 2014: 104-105).

19 Le monastère de Ximing fut construit entre 656 et 658 sur un décret de l'empereur Gaozong, et en plus d'un centre d'enseignement, il était également un centre de traduction de textes sanskrits. Xuanzang en personne y fut brièvement installé comme premier abbé après son retour d'Inde. Wonch'uk y travailla en étroite collaboration avec ce dernier de 656 à 658 environ et devint si étroitement associé au monastère qu'il est également connu sous le nom de « maître Ximing " Ximing fashi 西明法師. Tankuang, qui y résida un siècle après Xuanzang, à partir de l'année 735 environ, écrivit vraisemblablement plusieurs de ses compositions dans ce monastère. 
la capacité à l'éveil des êtres. Tandis que les exégètes Tathāgatagarbha défendent l'universalité de la capacité à l'éveil pour tous les êtres, Kuiji affirme des capacités différenciées à l'éveil en invoquant la doctrine des « Cinq lignées spirituelles » (wu zhongxing 五種性). ${ }^{20}$ L'accent mis sur cette doctrine par Kuiji commence à apparaître dans son commentaire au CWSL, dont la composition est quasiment simultanée à la rédaction de ce texte par Xuanzang.

Dans son commentaire, Kuiji décrit la doctrine des « Cinq lignées spirituelles » en détail, ${ }^{21}$ et y affirme l'existence d'êtres « dépourvus de lignée spirituelle » (wu zhongxing無種性, agotra) ou icchantika (yichanti 一闡提).22

Non seulement la doctrine des « Cinq lignées spirituelles » défendait une position diamétralement opposée à la théorie Tathāgatagarbha, mais elle était également en contradiction avec l'enseignement du Véhicule unique du Sūtra du Lotus et de la doctrine du Sūtra du Nirvāna, selon lequel tous les êtres, y compris les êtres "dépourvus de lignée spirituelle », deviennent des buddha. Ces sūtra étaient très en vogue en Chine du Nord à l'époque du retour en Chine de Xuanzang, et certains ont suggéré que l'école Faxiang a disparu de l'histoire du bouddhisme peu de temps après Xuanzang précisément à cause de la position radicale de l'école sur cette question. ${ }^{23}$

Nous savons que les positions doctinales de Wonch'uk étaient différentes de celles de Kuiji et de ses disciples, et selon certaines sources, il aurait même existé une rivalité continue entre Wonch'uk et Kuiji. ${ }^{24}$ Certains chercheurs japonais parlent de polarisation des sources concernant Wonch'uk. ${ }^{25}$ Selon eux, les idées de Wonch'uk auraient été rejetées parce

20 Cette théorie de classification des êtres sensibles en cinq catégories sur la base de leur potentiel à l'éveil, avec un groupe exclu de façon permanente du salut, fut établie en Chine par Xuanzang et ses disciples et est spécifique à l'école Faxiang. Cette idée était clairement héritée de Dharmapāla, puis enseignée par son disciple Sīlabhadra (ca. 529-645), qui fut le maître principal de Xuanzang au monastère de Nālandā, où il étudia durant cinq ans. Dharmapāla affirmait qu'il existait une catégorie de personnes pour qui l'état de buddha était impossible à atteindre. Ces êtres, bien que ce ne soit pas de leur faute, manquaient de façon inhérente de ce qu'il appelait « les germes non-parfumés » (ou « germes non-contaminés » wulou zhongzi 無漏 種子, ainsi que le traduisit Xuanzang) et étaient de ce fait éternellement exclus du salut. Selon la tradition, Xuanzuang aurait dans un premier temps hésité à introduire cette théorie en Chine, où la croyance en une Nature de Buddha inhérente était forte.

21 T.1831.610c19-611a01.

22 " êtres sans foi ", totalement dépourvus de semences de vertu susceptibles de les mener à l'Eveil. Exclus définifs de la bouddhéité ou sorte de « damnés » du bouddhisme (Cornu 2006: 267).

23 Weinstein 2009: 293-297.

24 Cf. la courte biographie de Wonch’uk dans le Song Gaoseng zhuan 宋高僧轉, Biographies des moines éminents de la Dynastie des Song, compilée par Zanning (919-1002), T. 2061.727b.

25 Jorgensen 2002: 74-133. 
qu'opposées à la position orthodoxe de l'école Faxiang, et certains l'on même qualifié d' " hérétique de l'école Faxiang ". ${ }^{26}$ Ses idées furent néanmoins perpétuées durant plusieurs générations dans son monastère de Ximing, où Tankuang en a probablement pris connaissance avant de les propager à Dunhuang.

La position doctrinale de Wonch'uk se trouvait probablement entre celles de Paramārtha et de Xuanzang, tout en favorisant les positions de Paramārtha au détriment de Xuanzang sur beaucoup de points doctrinaux. C'est cette préférence à l'encontre de Paramārtha, qui était en contradiction avec les autres savants de l'école Faxiang, qui engendra donc de sévères critiques à l'égard de Wonch'uk à son époque, et influença également la compréhension des vues des chercheurs modernes à l'égard de Wonch'uk.

Ainsi, en affirmant que même les icchantika peuvent être sauvés ou éveillés, Wonch'uk contredit la position de Xuanzang. Selon lui, la doctrine de l'icchantika serait apparue dans les sūtra comme un « moyen habile » (upāya $)^{27}$ pour motiver certaines personnes à faire plus d'efforts. Il est vrai, dit-il, que tout le monde possède de façon inhérente la Nature de Buddha (tathāgata); cependant, certains doivent remplir les conditions qui leur permettent de devenir éveillés. Il présente la dégradation extrême des icchantika comme une condition temporaire, qui peut être écartée à l'aide de pratiques religieuses adaptées. Sa position n'est donc ni celle défendue par le nouveau Yogācāra, ni tout à fait celle des défenseurs de la théorie tathāgatagarbha, puisqu'il fait de la Nature de Buddha inhérente à tous les êtres une condition potentielle, pouvant être atteinte au moyen de certaines pratiques.

Quant à Tankuang, il ne mentionne qu'une seule fois les icchantika dans son commentaire au $Q X L$, lorsqu'il évoque les sept sortes de gens qui ne peuvent entrer dans le samādhi ${ }^{28}$ de l'Ainsité (zhenru sanmei 真如三昧):

26 Kamata Shigeo, Chōsen buddyō no tera to rekishi (Tokyo: Daihōrinkaku, 1980), p.161. Cité par Jorgensen.

27 Upāya kausalya, en chinois fangbian 方便, traduit par " moyens habiles », " méthode », ou encore " expédient salvique », désigne dans le Mahāyāna tous les moyens mis à disposition des bouddha pour aider les êtres à s'arracher à la souffrance et atteindre l'Eveil. Ces moyens sont qualifiés de " habiles ", car ils sont adaptés en fonction des besoins et des capacités des êtres (Cornu 2006: 673-674).

28 " Recueillement », " absorbtion méditative ». Terme générique qui désigne tout état de méditation profond obtenu après la stabilisation de l'esprit, lorsque celui-ci demeure focalisé sur un seul point ou sur un objet unique, et que les notions de "sujet " et d' " objet " s'évanouissent. Cornu: 499. D’après le QXL: «... grâce à ce samādhi, on connaîtra le caractère d'unité de la sphère de la Loi. On entend par là que le corps de la Loi de tous les buddha est égal et non duel avec le corps des êtres. » (T.1666.582b01-13) et « Pour les profanes qui n’ont 
Premièrement, les sceptiques: les personnes dont la nature est de douter. Deuxièmement, les mécréants: les personnes incrédules (icchantika) dénuées de volonté. Troisièmement, les blasphémateurs: les non-bouddhistes (litt. en dehors de la Voie) qui enseignent des hérésies. Quatrièmement, les fauteurs graves: les personnes qui ont commis [des actes] tels que les dix mauvaises actions et dont l'esprit est impur. Cinquièmement, les criminels: les personnes [qui ont commis] les cinq péchés capitaux et dont l'esprit est extrêmement mauvais. Sixièmement, les orgueilleux: les personnes imbues d'elles-mêmes qui refusent d'apprendre. Septièmement, les paresseux: les personnes indisciplinées dont l'esprit est dissipé.

Aucun d'eux ne peut entrer immédiatement [dans le samādhi]. ${ }^{29}$

Ils ne le peuvent peut-être pas maintenant ni dans ces conditions, mais selon le $Q X L$, les profanes qui n'auraient pas assimilé la méthode de samādhi, ont encore une chance de parvenir au salut par la récitation des noms du Buddha et ainsi pénétrer dans les Terres pures. ${ }^{30}$

Tankuang semble également donner une chance de salut à ces êtres pour qui le samādhi est encore inaccessible. En effet, il affirme que le profane et le Saint ne sont pas différents dans leur essence. ${ }^{31}$ Il continue en expliquant que l'absence, chez certains êtres vivants, de la faculté à accomplir le bien est un obstacle temporaire. ${ }^{32}$

Enfin, Tankuang cite le « nouveau » Traité quant aux pratiques qu'il faut cultiver afin de pouvoir, en fin de compte, entrer dans le samādhi:

Devenir proche de tous les buddha et bodhisattva. Leur rendre hommage, leur faire des offrandes, les vénérer et les louer. Ecouter le Dharma correct et cultiver les pratiques telles qu'elles sont enseignées. ${ }^{33}$

pas assimilé la méthode de samādhi, l'entrée dans la famille des tathāgata n'a aucunement lieu d'être. » (T.1666.582b29-c01) Trad (Girard 2004).

29 Ma traduction de T.2814.1168a24-b01:... 七種人所不能入。一疑惑者。凡愚之人性猶豫故。 二不信者。闡提之人不希望故。三誹謗者。外道之人記邪見故。四重罪者。十惡等人心不淨 故。五業障者。五逆之人心極惡故。六我慢者。自高之人不屈學故。七解急者。放逸之人心縱 蕩故。隨有一者即不能入。新論合彼第四第五種為一種。但名業障。皆為業障所纏障故。

30 Cf. T.1666.583a16-21. Comme le note Girard, la version de Śikșānanda infléchit encore un peu plus ce passage dans la direction des laïcs en affirmant: « en cultivant graduellement les pratiques, il arrivera à entrer dans le stade de juste [détermination] » (Girard 2004: 163).

31 Cf. T.1814.1168b09: 凡聖同真無異體故。

32 Cf. T.1814.1168.b26: 此舉障因。謂無增上善根力。非謂常無善根力也。

33 Ma traduction de T.1667.591a29-b01: 親近一切諸佛菩薩。頂禮供飬恭敬讚歎。聽聞正法如 說修行。 
Tankuang paraît donc se rallier à la position de Wonch'uk, selon laquelle l'Eveil reste accessible à tous, à conditions d'observer certaines pratiques, plutôt que de condamner une partie des êtres à ne jamais connaître l'Eveil.

\section{La question de la $9^{\text {ème }}$ conscience}

Il est une autre question qui a suscité d'intenses débats entre les tenants d'un " ancien » Yogācāra et d'un « nouveau » Yogācāra, il s’agit de la question de la $9^{\text {ème }}$ conscience ou amalavijñāna.

La doctrine de l'amalavijñāna ou de la Neuvième Conscience (jiushi 九識) a traditionnellement été attribuée à Paramārtha. ${ }^{34}$ Il s'agit en fait d'un système Yogācāra typique de huit consciences auquel est ajouté une " conscience immaculée » (amoluo shi 阿摩羅識 ou wugou shi 無垢識, amalavijñāna), conçue comme une corrélation purifiée de la " conscience de tréfonds » (alaiye shi 阿賴耶識, ālayavijñāna). ${ }^{35}$

Xuanzang s’opposa à l'idée des neuf consciences de Paramārtha en plusieurs passages de ses propres écrits et ses disciples firent de même. Pour être précis, les exégètes du « nouveau » Yogācāra acceptent également la notion d'amalavijñāna, mais au contraire de Paramārtha qui la voit comme une $9^{\text {ème }}$ conscience séparée de la $8^{\text {ème }}$, ils la considèrent comme la partie pure de la $8^{\text {ème }}$ conscience -

34 L'attribution traditionnelle du système des neuf consciences à Paramārtha, cependant, est également controversée. Même si le concept de l'amalavijñāna apparaît chez Paramārtha, il n'existe, d'après Michael Radich, aucune mention du système des neuf consciences, ni de l'amalavijñāna en référence à la $9^{\text {ème }}$ conscience. Ce sont des exégètes plus tardifs qui mentionnent le système des neuf consciences et en attribuent la paternité à Paramārtha (Radich 2008: 104-114).

35 L'ālayavijñāna est concept propre à l'école Yogācāra, définissant le réceptacle des traces karmiques, qui supporte et nourrit les sept autres consciences. On peut le comparer à une sorte de psychisme subconscient et impersonnel, sur lequel reposent les six connaissances sensorielles, l'esprit (manas) et les objets connaissables. C'est la clef pour comprendre l'agir et le penser humain. Elle n'a pas d'existence continue, puisqu'elle se renouvelle à chaque instant sous l'effet des "germes" qui tiennent leur force des actes antérieurs et deviennent facteurs de projection du sujet dans le devenir. Ce renouvellement est si rapide que cette succession d'imprégnations donne l'illusion d'une continuité, d'un moi permanent, d'une identité de personne. Cf. May 1971: 308. Le terme est utilisé par Tankuang dans cette transcription, mais il est intéressant de noter qu'il utilise également (et il est le seul parmi les auteurs Faxiang à le faire) la translitération du sanskrit telle qu'utilisée par Paramārtha (liyeshi 黎耶識). 
l'ālayavijñāna - qui ne se manifeste pas avant le stade de la Terre du Tathāgata (rulai di 如來地). ${ }^{36}$ Ils n'établissent pas de $9^{\text {ème }}$ conscience séparée. ${ }^{37}$

Cette distinction semble être un des facteurs qui ont contribué à l'établissement de la bifurcation entre l' " ancien » et le " nouveau » Yogācāra, ou entre les traditions Tathāgatagarbha et Yogācāra. En effet, l'amalavijñāna prise comme $9^{\text {ème }}$ conscience implique que tous les êtres possèdent de façon innée la conscience immaculée, rejoignant ainsi la doctrine tathāgatagarbha.

Paramārtha associe l'amalavijñāna avec plusieurs concepts qu'on ne trouve pas dans le QXL et qui ont des connotations tathägatagarbha, tels que l' " esprit pur par nature » (zixing qingjing xin 自性清淨心) ${ }^{38}$ qui est recouvert par les afflictions du [monde] extérieur (客塵煩惱), ${ }^{39}$ " la Nature de la réalité parfaitement accomplie» (yuancheng shixing 圓成實性) ${ }^{40}$ ou encore l' « Eveil originel » (benjue 本覺).

A ce sujet, Wonch'uk semble à nouveau adopter une position intermédiaire. Selon lui, si quelqu'un affirme comme le fait Paramārtha, qu'il existe une

36 Tankuang affirme la même chose: 故云依菩薩地盡入如來地能離。 “C'est pourquoi il est dit que, après avoir parcouru les terres ultimes des bodhisattva, l'on pénètre dans la Terre du Tathāgata, et il est [alors] possible de se dégager [des souillures]. » Ma traduction de T.2814.1139c03.

37 Le CWSL affirme qu'avec ālayavijñāna, l'amalavijñāna est une des dénominations de la 8 ème conscience, et que ce nom s'applique seulement au stade de tathāgatagarbha. Il affirme que cette conscience immaculée est associée avec la Grande connaissance de miroir parfaite ( $d a$ yuanjing zhi 大圓鏡智, ādarśa-jñāna), une des quatre connaissances acquise par la transformation de la conscience souillée. (cf. T.1585.13c08-24). Commentant ce passage, Kuiji ajoute qu'il est erroné de considérer l'amalavijũāna comme la $9^{\text {ème }}$ conscience. (cf. T.1830.344c09-13). Chez Tankuang, la Grande connaissance de miroir parfaite est à l'origine des bons dharma chez les êtres: 大圓鏡智能起眾生諸善法影。何故唯現善法影耶。(T.2814.1128c24-28). Dans un autre passage, il semble que Tankuang considère (s’appuyant entre autre sur le Traité sur les Terres des Maîtres du Yoga) que " l'ālaya est pur, moralement neutre [c.à.d. sans influence sur le karma], et entièrement dépourvu de plaisir ou de souffrance. " Il mentionne également que chaque être est à moitié égaré et à moitié éveillé, ce qui pourrait indiquer une " potentialité » d'Eveil ou une nature de Buddha inérente à tous les êtres. Enfin, Tankuang affirme également que le stade de la réalisation correspond à la Connaissance de miroir, et que lorqu'elle est réalisée, [la conscience] est totalement pure. (T.2814.1133a28-b07).

38 自性清淨心。名為如來藏 (T.1844.208b07-08). Ailleurs, il est presque toujours la cause de la mise en mouvement du vent de l'ignorance (Cf. T.1844.209b10; 228a06; 231a11). Chez Tankuang: 心性無動即是自性清淨心義故。(1149b22) 《 La nature de l'esprit est dépourvue de mouvement, c'est justement le sens de l'esprit pur de par sa nature ».

39 Le terme est également cité par Tankuang, dans un passage qu'il tire probablement de Wonhyo (Cf. T.2814.1138b09-11, également cité en p. 21).

40 Terme également utilisé par Tankuang quelques lignes plus haut: 應言入圓成實。若入圓成 實性時。(T.2814.1136a05). 
neuvième conscience pure, alors il doit être admis que la $8^{\text {ème }}$ conscience, l'ālayavijñāna est impure. Wonch'uk se pose donc en faveur de l'idée que la $9^{\text {ème }}$ conscience n'est rien d'autre que l'aspect pur de l'ālayavijñāna, et que cet aspect pur de la $8^{\text {ème }}$ conscience ne doit pas être confondue avec l'existence d'une neuvième. Ainsi, Wonch'uk rejette l'idée de Paramārtha d'une $9^{\text {ème }}$ conscience et semble adopter la position des exégètes Yogācāra.

Cependant, concernant l'ālayavijñāna, Wonch'uk affirmait qu'elle contenait à la fois des qualités pures et impures - tout en étant pure dans sa vraie nature. La définition de Wonch'uk de l'ālayavijñāna comme étant " parfumée " ${ }^{41}$ mais néanmoins pure dans sa vraie nature différait donc de celle de Xuanzang, pour qui elle était seulement « parfumée ».

Il est une nouvelle fois difficile de connaître la position de Tankuang sur cette question. En effet, les termes « amalavijñāna " et " $9^{\text {ème }}$ conscience " n'apparaissent pas dans ses commentaires du QXL. De plus, bien qu'il en parle dans d'autres textes, il n'utilise cependant jamais la transcription " classique » du terme 阿摩羅識 (amoluoshi). A la place, il utilise des variantes de transcription qui n’apparaissent pas chez Paramārtha, telles que 阿末羅[識] (amoluoshi), ${ }^{42}$ ou 阿磨羅識 (amoluoshi). ${ }^{43}$

Selon Radich, lorsqu'un auteur ne respecte pas la transcription usuelle, cela peut nous indiquer, par exemple, qu'il a entendu un terme mais ne l'a pas lu; ou que la connaissance de ce dernier lui est parvenue par le biais d'une source différente que Paramārtha lui-même.

Il existe toute une variété d'autres termes se référant à l'amalavijñāna dans les sources plus tardives, qui n'étaient pas utilisés dans les textes de Paramārtha. Par exemple le terme de 無垢識 (wugoushi), qui est assimilée à la

41 « Le parfumage (vāsanā) est l'acquisition d'une seconde nature grâce à l'habitude, à l'expérience répétée d'un phénomène. Il s'entend en deux sens: la force active qui imprègne le psychisme; et la puissance passive latente qui reste dans le fond du psychisme. »Cf. Girard 2004: 64, note 133. Il sera question du parfumage de manière plus détaillée plus loin.

42 T.2810.1051b09-11: 三名阿末羅。此名無垢識。Cette transcription est également utilisée par Kuiji et un de ses collaborateur coréen (mais pas Wonch'uk!), ainsi que par Bukong et le disciple de ce dernier, Huilin, qui séjourna au monastère Ximing peu avant Tankuang. Elle est également utilisée par un grand nombre de moines japonais postérieurs à Tankuang, surtout de l'école Hosso (Faxiang japonais).

43 T.2812.1075a20 (cf. note 46 page suivante). Cette même transcription n'est utilisée que par deux moines qui vécurent avant lui, dont Kūkai 空海 (774-835), moine japonais qui introduisit l'école Shingon au Japon et qui résida à Chang'an entre 804 et 806. Ce dernier affirme que la $9^{\text {ème }}$ conscience, l'amalavijñāna correspond à la conscience naturellement pure des buddha: 謂 第九阿磨羅識即佛性淨識是也。(T.2701.361b29-C01). 
neuvième conscience et se trouve dans les écrits de plusieurs moines dès la fin du 6ème siècle, ${ }^{44}$ ou encore 淨識 (jingshi). ${ }^{45}$

Cependant, la variante la plus significative du terme amalavijñāna est « neuvième conscience » 九識 (jiushi). De nombreuses sources plus tardives expliquent fréquemment que Paramārtha aurait présenté l'amalavijñāna comme une 9ème conscience, qui venait se placer au-dessus du modèle habituel à huit consciences du Yogācāra " classique ». Des références à l'amalavijñāna en tant que " neuvième conscience » se retrouvent chez plusieurs auteurs dont Kuiji, Wonhyo et Tankuang. Ce dernier mentionne un Jiushi lun 九識論 de Paramārtha, en lien avec le Lankāvatārasūtra, selon lequel:

Le nom de la 9ème consience est amalavijñāna. Il s'agit de l'alāya qui s'est départi des souillures des naissances et des disparitions. C'est à dire l'Ainsité appelée amalavijñāna. ${ }^{46}$

Il est important de noter, comme le souligne Radich, que la première mention d'une neuvième conscience ou de neuf consciences, ne se trouve pas chez Paramārtha ou ses successeurs, mais dans le Lankāvatārasūtra. ${ }^{47}$

Les érudits tardifs se référaient fréquemment à ce passage, ou à sa doctrine, lorsqu'il était question du concept d'amalavijñāna et de la neuvième conscience, mais dans un sens qui tend à s'éloigner de celui que leur a donné Paramārtha. En effet, l'on peut constater qu'après Kuiji et Wonch'uk, la gamme des significations que nous rencontrons s'est significativement appauvrie et dépend la plupart du temps de l'interprétation des auteurs Faxiang qui les ont précédés. ${ }^{48}$

44 Le terme apparaît dans les deux textes de Tankuang déjà mentionnés plus haut: dans son T.2810, aux lignes 1051a12-14; 1051b09-11 (passage vu plus haut); 1055a26-28. Ainsi que dans son T.2812, aux lignes 1075b06-08 et 1075c12-14. Il n'apparaît cependant pas dans ses commentaires du QXL. Dans aucun de ces passages Tankuang ne mentionne qu'il s'agit d'une $9^{\text {ème }}$ conscience, il l'assimile plutôt à l'aspect pur de l'alāyavijñāna, la $8^{\text {ème }}$ conscience.

45 Ce terme apparaît également dans les deux mêmes travaux de Tankuang: le T.2810, au lignes 1054b24; 1055a08; 1055a15; 1054b24; etc., ainsi que dans le T.2812, dont deux fois dans le passage 1079b16-20. Là encore, Tankuang ne mentionne pas ce terme comme se référant à une $9^{\text {ème }}$ conscience, mais bien comme faisant partie de la $8^{\text {ème }}$.

46 T.2812.1075a18-21: 眞諦三藏譯九識論亦同楞伽。其前八識與此不殊。第九名爲阿磨羅識。 意説頼耶離生滅染。即是眞如名阿磨羅識。Ainsi que dans son T.2810, lignes 1010b21-22: 楞 伽經等開阿頼耶染淨二位説有九識。若攝大乘依前六識根境識三立十一識。Où il mentionne que dans le Lankāvatārasūtra il est question de 9 consciences.

47 "Les différentes consciences, de huit ou neuf sortes, sont telles des vagues sur l'eau », ma traduction de 八九種種識, 如水中諸波。(T.671.565b24), traduction chinoise de Bodhiruci.

48 Il ressort également de ces comparaisons que Wonch'uk suit en partie Kuiji dans son interprétation concernant l'amalavijñāna. D'autre part, tandis que amoluoshi 阿末羅識 n'est utilisé que par Kuiji, amoluoshi 阿摩羅識 est utilisé par Wonch'uk et Kuiji, mais ni par Wonhyo ni par Tankuang. 
Cela est valable partiellement pour Tankuang, qui utilise soit une transcription employée seulement par Kuiji ${ }^{49}$ (mais pas par Wonch'uk, ni Wonhyo) soit une transcription plus ancienne, utilisée par des auteurs qui ont séjourné à Ximing avant lui. Enfin, il mentionne une neuvième conscience, soit en référence à un Jiushi lun 九識論 dont Paramārtha serait l'auteur, soit en se référant au Lankāvatārasūtra.

Ce qui est donc marquant dans la position de Wonch'uk, et qui semble avoir également influencé Tankuang, est son attitude accomodante, que Lee qualifie de « middle way stand ». En effet, plutôt que de critiquer ses adversaires, Wonch'uk émet ses inquiétudes concernant la culture intellectuelle bouddhiste de son temps, spécialement en ce qui concerne le conflit entre les pensées Madhyamaka $^{50}$ et Yogācāra. Le fondement de la controverse se trouvait dans le fait que les Mādhyamika considéraient que tous les êtres étaient tathāgatagarbha tandis que les Yogācāra considéraient que tous les êtres ne possédaient pas la Nature de Buddha universelle.

Wonch'uk affirme que les enseignements de Dharmapāla doivent être compris depuis une perspective qui prend en considération le contexte qui donna naissance à chacun des enseignements. Ces maîtres ont produit des enseignements qui étaient adaptés à la situation de leur audience, de façon à ce que leur enseignement puisse permettre à tous les êtres de réaliser la Voie du milieu. Aussi longtemps qu'ils satisfont le but commun de l'enseignement bouddhiste qui consiste à permettre aux êtres de devenir éveillés, ils sont valides et équivalents les uns aux autres. Ainsi le fait de servir le but de sauver les êtres devient la fondation des principes que Wonch'uk va développer dans son érudition doctrinale.

\section{Influence de Wonhyo}

Toujours dans une perspective harmonisante, un autre moine a eu une grande influence dans cette controverse - et sans doute également sur Tankuang - en introduisant une sorte de synthèse de l' « ancien » et du « nouveau » Yogācāra,

49 Il est intéressant de noter que Tankuang mentionne les travaux de Kuiji à plusieurs reprises. Par exemple à la ligne T.1814.1123c22 il mentionne le Qijuan jing 七卷經 de Kuiji (T.1861), bien qu'il ne le cite pas. Plus loin, il cite exactement le commentaire du Traité du Milieu et des Extrême de Kuiji (T.1835) à deux reprises (lignes T.2814.1134a14 \& 1143b10), et enfin, une nouvelle fois une citation exacte du Traité en sept fascicules (T.2814.1150b14).

50 " La Voie du milieu ». Il s'agit de l'une des deux grandes écoles philosophiques du Mahāyāna avec le Yogācāra. 
prenant appui pour cela sur le QXL. Il s'agit de Wonhyo (617-686), un autre moine coréen dont les commentaires eurent une grande influence sur la tradition bouddhique dans toute l'Asie de l'est. Wonhyo s'intéressa tout particulièrement aux pensées du Tathāgatagarbha et Yogācāra, mais ses compositions et ses commentaires (il en composa plus de cent) couvrent tous les enseignements du bouddhisme Mahāyāna connus en Asie de l'est. Parmi ses écrits les plus influents figurent les commentaires qu'il fit sur le $Q X L{ }^{51}$ Ces derniers eurent une influence considérable sur les savants bouddhistes de Chine et du Japon, et participèrent à l'établissement de ce texte comme l'un des plus influents de la région. Bien qu'il n'ait pas séjourné en Chine, ses textes y ont circulé et y ont été largement étudiés et commentés.

Son approche a souvent été qualifiée par les chercheurs modernes de tentative d' " harmonisation des disputes ». En effet, l'un des principaux buts de Wonhyo semble avoir été de dépasser les conflits doctrinaux en utilisant de façon rigoureuse et logique leur contenu, afin de démontrer leur compatibilité de fond et parvenir ainsi à révéler un système global correspondant au bouddhisme Mahāyāna. Il semble en effet qu'à l'époque de Wonhyo et de Wonch'uk, surmonter la dispute entre les deux écoles rivales Madhyamaka et Yogācāra était un sérieux problème. Selon Wonhyo, cette dispute pouvait être harmonisée par la doctrine du QXL si cette dernière était bien comprise. Il affirme donc la suprématie du Traité sur les deux doctrines rivales entre elles. ${ }^{52}$

Le QXL est connu pour sa synthèse des systèmes Tathāgatagarbha et Yogācāra. L'affirmation contenue dans le Traité que le tathāgatagarbha et l'ālayavijñāna sont unis d'une manière qui n'est " ni identique ni différente » (feiyi feiyi 非一非異) ${ }^{53}$ était généralement considérée comme un indice de solution de la contradiction doctrinale entre les exégètes Madhyamaka et Yogācāra.

La thèse de l' "Esprit un et ses deux aspects » (yixin ermen 一心二門) du QXL contient une solution potentielle à la contradiction doctrinale entre Madhyamaka et Yogācāra, étant donné que les deux aspects de l'Esprit un, son « aspect en tant qu'Ainsité » (xin zhenru men 心真如門) et son « aspect soumis aux naissances et aux disparitions »(xin shengmie men 心生滅門), correspondaient respectivement aux domaines inconditionné et conditionné. ${ }^{54}$

51 T.1844 et T.1845. Dont une petite partie a été traduite en anglais par Park (1979).

52 Son commentaire du QXL englobe toutes les doctrines bouddhistes de façon compréhensive et d'une manière non sectaire, tandis que d'autres commentateurs, à l'image de Fazang (643712), tentèrent au contraire de s'approprier le Traité afin de glorifier leur propre école.

53 Tankuang affirme la même chose: 故覺不覺非一非異。 « C'est pourquoi l'Eveil et le nonEveil ne sont ni identiques ni différents. » (T.2814.1129b16-20).

54 Toutes les choses ou substances engendrées, c'est-à-dire constituées par la réunion de causes et de conditions, sont dites « composées » (samskṛta) ou " conditionnées » puisqu'elles 
De plus, le modèle graduel du tathāgatagarbha ressort également clairement de sa compréhension de l' "Esprit un ». Wonhyo affirme en effet que lorsqu'une personne atteint le «stade ultime » (jiujing wei 究竟位) après avoir éliminé les quatre marques, ${ }^{55}$ l'éveil initial ne se distingue pas de l'Eveil originel et l'Esprit retourne à son origine qui n'est autre que l" "Esprit un ". ${ }^{56}$ Autrement dit, ce n'est pas avant que le tathāgatagarbha (ou Eveil potentiel) devienne manifeste comme la forme complète d'éveil après un processus de pratique que l'esprit retourne à sa source. ${ }^{57}$

D'un côté, l' "Esprit un » est décrit comme existant toujours en même temps que tous les dharma; le QXL affirme qu'il englobe tous les dharma supramondains (chu shijian $f a$ 出世間法) et mondains (shijian $f a$ 世間法) ${ }^{58}$ et Wonhyo affirme aussi qu'il est « le domaine de la Loi unique » (yi fajie 法界, dharmadhātu). ${ }^{59}$ De l'autre côté, l' « Esprit un » ne peut être obtenu avant

dépendent de causes et d'effets. Tout est composé, à l'exception d'une partie de l'élément des phénomènes mentaux et de leur source (Cornu 2006: 145). Tankuang, à la fin de son commentaire, donne une définition de ces deux aspects de l'esprit: le premier correspondant à ce qui ne peut être mesuré, le second à son aspect empirique. Lorsque les deux s'accordent, alors l'Esprit un est sous contrôle. Enfin, l'Esprit un renferme les deux aspects. (cf. T.2814.1173c27-29).

55 Les quatre marques de l'esprit; celle de la naissance (shengxiang 生相), de la durée (zhuxiang 住相), du changement (yixiang 異相) et de la disparition (miexiang 滅相). Girard parle de «phases temporelles » de la pensée (Girard 2004: 35).

56 Chez Tankuang aussi, les êtres, après s'être soustraits à des pratiques durant un temps incalculable, bénéficient de la puissance du parfumage et l'ignorance disparaît. Avec l'extinction de l'ignorance, son origine même s'en trouve complètement épuisée: 由前因中三無數劫久 遠熏力真用極顯至究竟位。無明即滅。無明滅者。根本無明盡也。(T. 2814.1144c20) Ensuite, toujours selon Tankuang, l'esprit de buddha, libéré des illusions, retourne à la source de l'Esprit un et l'Eveil initial est identique à l'Eveil originel, qualifié de « complet»: 佛心離妄歸一心源。始覺同 本名真實。(T.2814.1164a15) L'affirmation que l'Eveil initial n'est pas différent de l'Eveil originel revient plusieurs fois chez Tankuang et est également qualifié de « pur » (cf. lignes T.2814.1121c22; 1121c28; 1126b22).

57 En fait, à côté de l' "Esprit un et ses deux aspects ", Wonhyo utilise également une phrase « les deux aspects de l'Esprit un » (ermen yixin 二門一心) qui n'apparaît pas dans le QXL: 合則 二門一心之法為要 (T.1844.202b18-19), et selon Lee, il semble que cette expression représente le

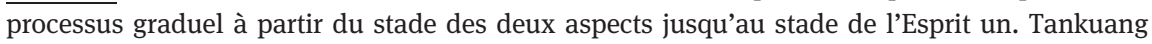
utilise une expression similaire: 既此二門唯是一心。是故止觀不相捨離。(T.2814.1166c29).

58 Cf. T.1666.575c20-25.

59 一心即是一法界故。此一法界通攝二門。而今不取別相之門。於中但取總相法門

(T.1844.207a24-26). Il est intéressant de noter qu'ici Tankuang cite le " nouveau » Traité pour expliquer que sans éveil dans le domaine de la Loi unique il n'y a pas d'association, faisant surgir l'ignorance et les discriminations: 故新論云。不覺一法界故不相應。無明分別起。(T.2814.1138c12) Enfin, Tankuang cite une nouvelle fois le «nouveau » Traité, qui affirme que tous les êtres et toutes les choses (dharma) appartiennent à la même sphère de la Loi unique et qu'il n'y en a pas deux.: 新 論云。一切眾生一切諸法。皆同一法界無有二相。(T.2814.1159a09-10). 
la fin d'un processus de pratique. Il comporte donc également une double signification à la fois de changement et d'immuabilité. ${ }^{60}$ L'Esprit un accepte aussi bien l'impermanence et le changement précisément parce qu'il englobe tous les dharma, et Wonhyo affirme aussi que l' " aspect de naissance et de disparition » (shengmie 生滅) et l' « aspect immuable » (changzhu 常住) ${ }^{61}$ ne se gênent pas l'un l'autre. Cet état ultime d'harmonie de l'Esprit un est attribué par Wonhyo à l'enseignement de l'Avatamsakasūtra. ${ }^{62}$

Dans le système de Wonhyo, avec lequel Tankuang semble être en accord, le tathāgatagarbha est donc expliqué comme un Eveil potentiel, qui se manifeste à travers un processus graduel de pratique, et c'est en réalisant l'Eveil parfait que l'on se rend compte que l'Eveil potentiel n'est pas différent de l'Eveil parfait.

La compréhension duelle du tathāgatagarbha par Wonhyo est également reflétée dans son explication des concepts de "non-éveil " (bujue 覺), ${ }^{63}$ d'" éveil initial » (shijue 始覺), ${ }^{64}$ et d' " éveil originel » (benjue 本覺). ${ }^{65}$ L'esprit, lorsque qu'il est non-éveillé du fait de l'emprise qu'exercent sur lui les illusions, transmigre parmi les Six destinées (liuqu 六趣).${ }^{66}$ Mais lorsqu'il est parfumé ( $x u n x i$ 熏習, vāsanā) par l'Eveil originel, l'esprit commence à rechercher le nirvāṇa à travers le processus de l'éveil initial jusqu'à ce qu'il retrouve l'Eveil originel et réalise que l'esprit n'a jamais changé ou été trompé. ${ }^{67}$

60 Cf. Lee 2014: 175.

61 Ces termes apparaissent un grand nombre de fois en l'espace de quelques lignes dans le commentaire de Tankuang (cf. lignes T.2814.1124c06-21), passage qui comprend également une citation presque exacte du commentaire de Wonhyo, qui cite lui-même le Mahāyānasaṃgraha (ou Somme du Grand-Véhicule).

62 Il est en cela suivi par Tankuang, qui le cite une nouvelle fois presque mot à mot (y compris le texte qui introduit la citation du Sūtra de l'Ornementation fleurie). Cf. T.2814.1124c20-27.

63 L'éveil des êtres qui n'ont pas (encore) éradiqué les racines du mal. Ils en sont au premier stade de l'éveil initial.

64 L'éveil initial (ou acquis), qui surgit de l'éveil originel grâce à l'imprégnation de l'esprit par l'Ainsité et grâce à l'enseignement bouddhique.

65 L'éveil originel (ou inné). Il s'agit de l'esprit éveillé des êtres, et se présente sous deux aspects: un éveil inconditionné, jamais souillé par l'ignorance et un éveil conditionné sujet à l'ignorance. L'Ainsité (zhenru 真如, tathāta) est l'essence ou la substance de l'esprit, et l'éveil originel en est la marque ou l'empreinte (相, laksana).

66 Idem chez Tankuang. Cf. lignes T.2814.1141a03: 是舉無明所迷法性。無明不覺妄與法違 者。à 1142b02-03: 此初也。染法不斷故有有漏六趣凡夫。淨法不断故有無漏三乘聖者。et suivantes.

67 Cf. T.1844.209c18-23. Tankuang parle également de cette aspiration au Nirvāna, citant le Mahāparinirvāna-sūtra: 即釋經中由有如來藏能厭生死樂求涅槃樂。涅槃經云。未來佛性力因緣 故還生善報。本覺內熏名佛性力。良以一識含此二義。(T.2814.1142c04-06) Il s'agit d'une citation presque exacte du sūtra original (T.374.570c24 \& T.375.818b22). Passage qui est également 
L'explication de Wonhyo sur les quatre étapes de l'éveil initial montre également sa compréhension duelle du tathāgatagarbha. Wonhyo divise le niveau de l'éveil initial en quatre étapes en associations avec les « quatre marques » (sixiang 四相), qui sont la naissance (sheng 生), l'existence (zhu 住), le changement ( $y i$ 異) et la disparition (mie 滅), c'est-à-dire les modes de conscience de quatre groupes d'êtres sensibles se trouvant à des étapes sotériologiques particulières. ${ }^{68}$ Chacune des quatre marques peut être éliminée en atteignant un niveau particulier de l'éveil initial dans une étape sotériologique correspondante. ${ }^{69}$ A la question de savoir si les quatre marques se produisent simultanément ou successivement, Wonhyo répond que ces quatre marques sont à la fois simultanées et successive d'une manière un peu paradoxale; il explique qu'elles sont simultanées une fois éveillé, mais successives avant l'éveil. ${ }^{70}$

La division par Wonhyo de l'éveil originel en deux types; " l'éveil originel afférent aux souillures » (suiran benjue 隨染本覺) ${ }^{71}$ et « l'éveil originel naturellement pur» (xingjing benjue 性淨本覺 $)^{72}$ peut également être compris à partir

cité dans deux écrits de Wonhyo: « Doctrinal Essentials of the Lotus Sutra » (T.1725.871a08) et " Doctrinal Essentials of the Nirvāna Sūtra » (T.1769.243a19), et dont a pu s'inspirer Tankuang. 68 Cf. T.1844.209c25-210c08. Tankuang semble donner une explication similaire (cf. T.2814.1122a10-14 et T.2814.1124b08-09).

69 Wonhyo explique que ces quatre marques sont éliminées en atteignant respectivement les quatre niveaux correspondants de l'Eveil initial, c'est-à-dire l' « Eveil ultime » (jiujing jue 究竟 覺), « Eveil partiel » (suifen jue 隨分覺), « Eveil en apparence » (xiangsi jue 相似覺), et «nonEveil » (bujue 不覺) (T.1844.209c25-210c08). On retrouve une classification similaire chez Tankuang; pour ce dernier, le " non-Eveil » correspond au niveau des profanes qui subissent la transmigration (T.2814.1123b07); ensuite, il qualifie l' "Eveil en apparence » de deuxième marque d'Eveil, celle des adeptes des deux Véhicules ayant le savoir d'examen mental, et qui sons donc capable d'Eveil (T.2814.1123c26-27); puis l' « Eveil partiel » en $3^{\text {ème }}$ marque d'Eveil, celle des bodhisattva ayant réalisé le Corps de la Loi, ce qui correspond à la $1^{\text {ère }}$ des dix terres de bodhisattva qui mènent à l'Eveil parfait, T.2814.1124a15-18; et enfin, l' "Eveil ultime » est atteint, selon Tankuang, lorsque l'on parvient à l'état de Tathāgata. (T.2814.1124c13).

70 Cf. T.1844.209a23-b02. Ceci est constitue une évolution par rapport au QXL, qui ne parle que de la simultanéité des quatre marques (T.1667.576c). Tankuang ne parle également que de la simultanéité des quatre marques. Pour lui, les quatre marques de l'éveil correspondent à l'Eveil Initial, mais ce dernier n'est, au final, pas différent de l'Eveil originel (T.2814.1125b29-c05).

71 Pour Tankuang, les aspects de naissance et de disparition se trouvent au centre de l'« éveil originel afférent aux souillures », mais ont une essence propre (T.2814.1126a18). Lorsque l' esprit de « éveil originel afférent aux souillures » retourne à [son] origine qui est la connaissance pure, l'Eveil initial est identique à l'Eveil originel, et c'est la raison pour laquelle on dit qu'il est pur. (T.2814.1126b20-22).

72 Wonhyo affirme que l'Ainsité qui devient parfumé se réfère à l' " Eveil originel naturellement pur » (xingjing benjue 性淨本覺). Etant donné que l'Eveil originel est un autre nom pour le 
de cette perspective duelle. Ces deux types d'éveil originel apparaissent pour la première fois dans son Qinxinlun shu 起信論疏, ${ }^{73}$ et ensuite Fazang et Tankuang reprendront la terminologie de Wonhyo. ${ }^{74}$

Etant donné que l' " éveil originel afférent aux souillures » est lié au monde phénoménal, on peut dire qu'il correspond à l'aspect changeant du tathāgatagarbha qui est relié à l'ālayavijñāna; étant donné que l' " éveil originel naturellement pur " se réfère à la qualité de base même de l'éveil; il semble correspondre à l'aspect immuable du tathāgatagarbha. De cette manière, Wonhyo relie ces deux types d'éveil originel respectivement à l'éveil initial et à l'éveil originel et il les relie aussi au Corps de rétribution et au Corps de la Loi. ${ }^{75}$

Le QXL quant à lui, décrit le tathāgatagarbha comme le substrat immuable de tous les phénomènes en le comparant à l'océan duquel surgissent d'innombrables vagues, ${ }^{76}$ et le qualifie également de Loi (Dharma) de l'Ainsité (zhenru fa 眞如法), qui est inhérent à tous les êtres et les incite à rechercher le nirvāna lorsque survient le parfumage venant de l'Ainsité (zhenru xunxi 真如熏習). ${ }^{77}$

C'est justement à travers le concept de « Parfumage par l'Ainsité » que Wonhyo explique le processus par lequel le tathāgatagarbha se développe en

tathāgatagarbha, Lee en déduit que Wonhyo relie le tathāgatagarbha avec la notion d'Ainsité. Tankuang quant à lui semble dire en substance que la Nature de buddha (ou tathāgatagarbha) n'est rien d'autre que l' « Eveil originel pur de par sa nature »: 四約佛性。此四既是性淨本覺。 (T.2814.1127c05).

73 T.1844.

74 Fazang l'utilise dans ces deux commentaires du QXL (T.1846 \& T.1847) et Tankuang fait de même dans ses commentaires (T.2813 \& T.2814).

75 (T.1844.211c21-25). Tankuang semble faire de même dans son commentaire (cf. lignes T.2814.1126b21 et 1148a25).

76 如大海水因風波動, 水相風相不相捨離, 而水非動性, 若風止滅動相則滅, 濕性不壞故。 (T.1666.576c09-13). « Ainsi en est-il de l'eau d'un grand océan agité par les vagues sous l'effet du vent. Le caractère de l'eau et celui du vent ne sont pas à part l'un de l'autre: l'eau n'ayant pas de mouvement par nature, lorsque le vent tombe, le caractère du mouvement disparaît, sans pour autant que celui de l'humidité soit détruit. " Trad (Girard 2004: 37).

77 真如熏習義有二種。云何為二? 一者、自體相熏習, 二者、用熏習。(...) 依此二義恒常熏 習, 以有力故, 能令眾生厭生死苦、樂求涅槃, 自信己身有真如法, 發心修行。

(T.1666.578b19-24). Passage que Girard traduit de la manière suivante: « Il est deux significations attachées au parfumage venant de la Talité. Quelles sont-elles? Premièrement, le parfumage dans l'être propre et les caractères de la Talité. Deuxièmement, le parfumage dû à son action. (...) Grâce à ces deux dernières significations, la Talité parfume en permanence. En raison de sa puissance, elle peut faire détester aux êtres la douleur des naissances et des morts et aspirer au nirvāna, et, en leur donnant créance au fait qu'ils ont en eux-mêmes le principe de la Talité, faire en sorte qu'ils conçoivent la pensée d'éveil et cultivent les pratiques. » (Girard 2004: 71). 
un éveil complètement manifesté. ${ }^{78}$ Alors qu'il explique le passage du QXL sur l' " éveil original afférent aux souillures », Wonhyo affirme que sur la base de la force de l'Ainsité qui parfume à l'intérieur (neixun 內熏), ${ }^{79}$ une personne peut pratiquer au « stade d'accumulation [des bonnes racines] » (ziliang wei 資糧位) ${ }^{80}$ puis au stade des bodhisattva, jusqu'à ce que le pratiquant atteigne le stade de l'éveil parfait (ou non-souillé) (wulou di 無垢地, dengjue 等覺), ${ }^{81}$ révélant ainsi la nature dépourvue de naissance et de disparition ${ }^{82}$; au contraire, dans le cas du 《Parfumage par l'Ignorance » (wuming xunxi 無明熏習), par lequel l'Ignorance parfume l'Ainsité, naît l'esprit troublé rendant tous les êtres prisonniers des naissances et disparitions. ${ }^{83}$

Ce qui est important dans l'explication de Wonhyo du parfumage par l'Ainsité ou par l'Ignorance est que ces parfumages prennent l'aspect d'un processus graduel à l'égard de l'éveil ou du saṃsāra.

C'est donc à travers son approche duelle que Wonhyo résout le conflit doctrinal entre Madhyamaka et Yogācāra. Sa compréhension du tathāgatagarbha comme la capacité universelle à l'Eveil inhérente à tous les êtres vivants correspond à la vue propre au Mahāyāna d'absence de distinction entre les deux mondes. Wonhyo se distingue néanmoins des Mādhyamika en ajoutant une étape entre la Nature de Buddha non-manifestée inhérente à tous les êtres et la Nature de Buddha achevée, obtenue au moyen de pratiques spécifiques.

Ainsi, la perspective adoptée par Wonhyo n'est pas non plus exactement identique à celle du Yogācāra car il accepte la notion de Nature de Buddha universelle qui, bien qu'initialement potentielle uniquement, peut être développée en un éveil complet, tandis que le Yogācāra la rejette.

Finalement, lorsque le tathāgatagarbha se manifeste comme la forme complète d'éveil à travers un processus de pratique, l'Esprit un, l'harmonie ultime de toutes les sortes de distinctions, est retrouvée. De cette façon,

78 Il semble qu'il en soit de même chez Tankuang, qui affirme que sans parfumage il est impossible d'accéder à l'état de buddha: 若殘習氣。成佛方斷。(T.2814.1139a18-19).

79 Parfumage interieur (neixun 內熏) ou mystérieux (mingxun 冥熏). Les deux termes aparaissent chez Tankuang.

80 Apparaît également une fois dans le commentaire de Tankuang (cf. T.2814.1144c02).

81 Tankuang mentionne également de l'accession au stade de la connaissance intuitive, qu'il associe au stade d'Eveil parfait du diamant: 初句總標能觀之位。即是金剛等覺之位。 (T.2814.1124b07).

82 Pour Tankuang c'est grace aux moyens habiles qu'il est possible de briser l'unité des manifestations de naissance et de disparition à l'intérieur de l'ālayavijñāna. (T.1844.211a14-18). 83 Selon Tankuang aussi, le parfumage par l'ignorance est la cause des illusions de l'esprit (cf. lignes T.2814.1138a09-11). 
Wonhyo accepte les deux positions, Madhyamaka et Yogācāra sans aucune contradiction dans la rubrique de l'Esprit un du QXL et ses deux aspects. La position de Wonhyo n'est donc pas une interprétation typique du $Q X L$, mais présente une nouvelle perspective tathāgatagarbha. ${ }^{84}$

Enfin, pour revenir à la question de l'amalavijñāna ou Neuvième conscience, Wonhyo identifie la notion d'Eveil originel du QXL au concept d'amalavijñāna de Paramārtha. ${ }^{85}$

Wonhyo affirme également que l'amalavijñāna est la $9^{\text {ème }}$ conscience, ${ }^{86}$ ce qui implique qu'il adopte une théorie de la conscience distincte de la théorie des huit consciences typiques de l'école Yogācāra et se différencie également de Tankuang sur ce point. Wonhyo considère l'amalavijñāna comme la $9^{\text {ème }}$ conscience, une conscience séparée de la $8^{\text {ème }}$ conscience, tandis que le Yogācāra la considère, comme nous l'avons vu, seulement comme la partie pure de l'ālayavijñāna, qui est atteinte lorsque l'on parvient à la Terre de Tathāta (rulai di 如來地).

Finalement, on observe d'autres similarités textuelles qui semblent appuyer l'hypothèse selon laquelle Tankuang connaissait très bien les écrits de Wonhyo, et en particulier ses commentaires du $Q X L$, et qu'il s'en est servi pour rédiger ses propres commentaires. Voici celles qui m’ont paru les plus évidentes.

\section{Similarités textuelles}

Nous avons déjà vu plus haut que Tankuang utilise parfois les mêmes citations de sūtra que Wonhyo, voici un exemple de citation du Lan்kâvatāra-sūtra ${ }^{87}$ par Tankuang:

\section{故楞伽云。身資生住持如夢中生。 ${ }^{88}$}

Cette dernière est presque en tout point exacte à celle du même texte par Wonhyo:

84 L'interprétation du QXL selon Wonhyo est tirée principalement de l'étude de Lee 2014.

85 令彼眾生皆得本覺者。是釋所化轉入之句。本覺正是唵摩羅識 (T.1730.978a19-20).

86 Cf. T.1730.978a06-08.

87 Il existe plusieurs traductions chinoises du sūtra, dont trois ont survécu; une traduction partielle de Gunabhadra datant du milieu du $5^{\text {ème }}$ siècle, une traduction en 10 fascicules de Bodhiruci datant de 513 et une traduction en sept fascicules de Śikșānanda datant de 700.

88 T.2814.1135c04. Trad.: « C'est pourquoi le Lañkâvatāra-sūtra dit: « Le corps et les richesses, la naissance et le maintien dans l'existence, se produisent comme dans un rêve. "» 
法非有之義如士卷經言身資生住持若如夢中生應有二種心而... ${ }^{89}$

Wonhyo précise qu’il cite le Lanka en 10 fascicules: “如十卷經 ».9

Cela est particulièrement intéressant, puisqu'à l'époque de Tankuang il existe une nouvelle traduction de ce texte, effectuée par Siiksānanda. Or, lorsque Tankuang cite directement le Lañkâvatāra-sūtra, sans s'appuyer sur des sources intermédiaires, il le fait dans la version de Śikṣānanda. Par exemple:

故楞伽云。謂若分別無我有二。是虚妄念。若如實知。彼念不縁名縁眞禪。91

qui est une citation presque exacte de la traduction de Śikṣānanda:

何攀縁眞如禪 謂若分別無我有二是虚妄念若如實知彼念不起是名攀縁眞如禪...92

Voici encore un autre exemple intéressant d'une citation du Śrīmālādevī-sūtra ${ }^{93}$ ainsi que du Lañkâvatāra-sūtra ${ }^{94}$ :

... 故勝䯭云。自性清淨心難可了知。彼心為煩惱所染。亦難可了知。楞伽經云。如來藏是 清淨相。客塵煩惱垢染不淨。95

Non seulement ces textes sont également cités par Wonhyo:

如夫人經 $^{96}$ 言。自性清淨心。難可了知。彼心為煩惱所染。亦難可了知。楞伽經言。以如 來藏是清淨相。客塵煩惱垢染不淨。97

mais ils le sont de manière quasi identique, à nouveau d'après la traduction de Bodhiruci du Lañkâvatāra-sūtra.

89 T.1844.202a25. Citation qui n'est d'ailleurs pas très proche du texte original. Cf. T.671.523c10. 90 Celui de Bodhiruci, traduit au $6^{\text {ème }}$ siècle est en dix fascicules.

91 T.2814.1124a29-b02. Trad.: Le Lañkâvatāra-sūtra dit: "Si l'on distingue deux non-moi dénués de toute pensée fictive, si la connaissance des choses "telles qu'elles » agit en sorte que les pensées [fallacieuses] ne surgissent pas, alors on appelle cela le dhyāna sur les conditions de l'authenticité. »

92 Cf. T.672.602a04-23.

93 Cf. T.353.222c02.

94 Cf. T.671.556b16.

95 T.2814.1138b09-11. Trad.: C'est pourquoi le Śrīmālā dit: « La pensée pure par sa nature est difficile à comprendre. » [Le fait que] l'esprit est souillé par les afflictions est également difficile à comprendre. Le Lankkâvatāra-sūtra dit: "Le tathāgatagarbha est la maque de pureté. Les afflictions [du monde] extérieur sont des pollutions non-pures. »

96 autre nom pour le Śrīmālā-sūtra.

97 T.1844.214c10-12. 
Il existe de nombreux exemples analogues avec d'autres sūtra, par exemple le Sūtra des Rois bienveillants (Renwang jing 仁王經), texte très en vogue à l'époque de Tankuang:

\section{故依此義復說偈云。伏忍聖胎三十人。十信十止十堅心等。98}

Il s'agit ici d'une citation exacte de la traduction de Kumārajīva, ${ }^{99}$ mais également des deux commentaires de Wonhyo, ${ }^{100}$ alors que Tankuang aurait à nouveau pu citer ce texte à travers une traduction plus récente, celle de Bukong. ${ }^{101}$

On peut encore dire la même chose d'une citation de la Somme du Grand Véhicule $^{102}$ :

故梁攝論顯中義云。能成立者。謂真本有十種功德。所成立者。謂則新生十種勝行。

Là encore, il pourrait s'agir d'une citation approximative du commentaire de Wonhyo:

染成於始淨故梁攝論云能成立者謂眞如十種功徳法所成立者謂十種新生正行也三約用熏故 應機成淨縁也染因名無明者謂六染... ${ }^{103}$

qui paraphrase lui-même la traduction de ce texte par Paramārtha, ${ }^{104}$ alors qu'il en existe également une traduction plus récente par Xuanzang!

On trouve un grand nombre d'exemples similaires dans le commentaire de Tankuang, avec des passages ou citations identiques ou proches des commentaires de Wonhyo. Ces passages se retrouvent parfois également dans les commentaire de Fazang, dont l'influence par Wonhyo est avérée. ${ }^{105}$

98 T.2814.1158b04. Trad.: « C'est pourquoi, sur la base de cette interprétation, une stance [du Sūtra des Rois bienveillants] parle de ceux qui maîtrisent les trente règles du contrôle de soi, et dont l'esprit [produit] les dix [pensées] de foi, les dix pratiques, les dix états d'esprit inébranlables, etc. »

99 Cf. T.245.827b08.

100 Cf. T.1844.202a25 et T.1845.237a07.

101 Cf. T.246.837b07. Il est intéressant de noter que le disciple de Bukong (ou Amoghavajra), Huilin (737-820), résida au monastère de Ximing à peu près en même temps que Tankuang.

102 T.2814.1149b08-10. Trad.: C'est pourquoi la Somme du Grand Véhicule des Liang, en explique la signification centrale disant: « Ce qui est démontré: le fait que les racines à l'origine comporte dix sortes de mérites. Ce qui doit être démontré: le fait qu'elles produisent de ce fait les dix sortes de pratiques supérieures (c'est à dire les dix pāramitā). »

103 Cf. T.1846.270a16.

104 Cf. T.1595.228c27.

105 Cf. Muller and Cuong 2012. 


\section{Conclusion}

Il semble assez clair que Tankuang a été influencé par Wonch'uk, en particulier dans sa vision harmonisante entre les différents courants (qu'ils soient considérés comme orthodoxes ou non), mais également sur la question de la $9^{\text {ème }}$ conscience. En effet, Tankuang semble suivre la position intermédiaire entre la théorie tathāgatagarbha et la position très stricte du courant défendu par Kuiji en admettant que l'alāyavijñāna comporte à la fois un aspect pur et un aspect qui ne l'est pas. De même, il semble également d'accord avec l'idée d'un processus graduel permettant d'atteindre l'Eveil, et potentiellement accessible à tous les êtres, qui possèdent tous une Nature de Buddha universelle, qui soit cependant être " activée » au moyen des pratiques adaptées.

Cependant, il faut noter qu'il lui arrive également de reprendre la terminologie de Kuiji. D’ailleurs, bien que Wonch'uk soit en désaccord sur de nombreux points avec Kuiji, il semble qu'il demeure largement lié au lignage Faxiang de Xuanzang, en particulier en ce qui concerne leur désaccord avec la théorie tathāgatagarbha du QXL.

$\mathrm{Au}$ contraire, Wonhyo, dont la pensée s'accorde avec la théorie du $Q X L$, représente une lignée différente, et le fait que Tankuang semble souvent en accord avec lui et qu'il s'est visiblement largement inspiré de ses commentaires a des implications importantes. En effet, cela voudrait dire que Tankuang a pu, d'une certaine manière, réconcilier deux positions très différentes, en particulier vis à vis du $Q X L$, en s'inspirant à la fois des enseignements de Wonch'uk et des écrits de Wonhyo.

Enfin, bien qu'à ce stade de mes recherches il s'agisse encore largement d'hypothèses qui ont besoin d'être vérifiées et appuyées par plus d'exemples, il semble néanmoins clair que Tankuang, qui reste encore largement méconnu et peu étudié, peut nous apporter une nouvelle perspective sur le bouddhisme Yogācāra qui a eu cours en Chine dans le courant du $8^{\text {ème }}$ siècle. Il paraît évident, à la lumière des similarités textuelles présentées dans le présent article, que Tankuang connaissait parfaitement les écrits de Wonhyo, même si ses positions quant à sa théorie du QXL ainsi que vis-à-vis des controverses de son époque ne peuvent être clairement établie sur cette base. C'est pourquoi, une étude plus approfondie des textes de Tankuang est nécessaire. Peut-être cela pourra-t-il également nous apporter des éléments de réponses quand à la disparition soudaine du courant Faxiang en Chine après la révolte d'An Lushan, alors que ce courant a continué d'exister au Japon jusqu'à nos jours. 


\section{Bibliographie}

Cho, Eunsu (2005): «Wonch'uk's Place in the East Asian Buddhist Tradition ». In: Currents and Countercurrents. Korean Influences on the East Asian Buddhist Traditions. Edited by E. Buswell. Honolulu: University of Hawai'i Press.

Cornu, Philippe (2006): Dictionnaire encyclopédique du bouddhisme. Paris: Editions Seuil.

Girard, Frédéric (2004): Traité sur l'acte de foi dans le Grand Véhicule. Tokyo: Keio University Press.

Jorgensen, John (2002): « Reprensenting Wonch'uk - Meditations on Medieval East Asian Buddhist Biographies ». In: Religion and Biography in China and Tibet. Edited by Benjamin Penny. Richmond: Curzon, 74-131.

Keng, Ching (2009): Yogacara Buddhism Transmitted or Transformed? Paramartha (499-569 CE) and His Chinese Interpreters. PhD Dissertation, Cambridge: Harvard University.

Lee, Sumi (2014): Toward a New Paradigm of East Asian Yogācāra Buddhism: Taehyon (ca. 8th century (E), a Korean Yogācāra monk, and His Predecessors. PhD Dissertation, Los Angeles: University of California.

May, Jacques (1971): « La philosophie bouddhique idéaliste ». In: Etudes asiatiques 25. Bern: Francke Verlag, 265-323.

Muller, A. Charles / Cuong, T. Nguyen (eds.) (2012): Wonhyo's Philosophy of Mind. Honolulu: University of Hawai'i Press.

Park, Sung Bae (1979): Wonhyo's Commentaries on the Awakening of Faith in Mahayana, Berkeley. Ph.D. Dissertation, Los Angeles: University of California.

Paul, Diana Y. (1984): Philosophy of Mind in 6th Century China: Paramārtha's Evolution of Consciousness. Standford: Standford University Press.

Radich, Michael (2008): « The Doctrine of *Amalavijnana in Paramartha (499-569), and Later Authors to Approximately 800 C.E. » ZINBUN 人文 41: 45-174.

Weinstein, Stanley (2009): Buddhism under the T'ang. New York: Cambridge Studies in Chinese History, Cambridge University Press. 